\title{
Potential of miR-25-3p in protection of chondrocytes: emphasis on osteoarthritis
}

\author{
Xiao He${ }^{1}$, Lili Deng ${ }^{2}$ \\ ${ }^{1}$ The Joint Surgical Center, Chenzhou No. 1 People's Hospital, Chenzhou, Hunan 423000, China \\ ${ }^{2}$ Pediatric Intensive Care Unit, Chenzhou No. 1 People's Hospital, Chenzhou, Hunan 423000, China
}

\begin{abstract}
Introduction. Osteoarthritis (OA) is the most prevailing musculoskeletal dysfunction triggered by lesions in synovial membranes and articular cartilage. MicroRNAs (miRNAs) have emerged as crucial regulators participated in many biological processes, such as osteoarthritis. This study was undertaken to address the role of miR-25-3p in the apoptosis of rat chondrocytes under an OA-like condition and its underlying mechanism.

Material and methods. OA cellular model was established in rat chondrocytes by TNF- $\alpha$ induction. Then, qRT-PCR and Western blotting were utilized for evaluation of the expressions of miR-25-3p and insulin-like growth factor-binding protein 7 (IGFBP7), CCK-8 assay for inspection of chondrocyte viability, flow cytometry for assessment of cell apoptosis rate, Western blotting for the detection of cleaved caspase-3 level and dual-luciferase reporter gene assay for verification of the targeting relationship between miR-25-3p and IGFBP7.

Results. The miR-25-3p expression was decreased and IGFBP7 was elevated in TNF- $\alpha$-induced rat chondrocytes. The miR-25-3p inhibited chondrocyte apoptosis and IGFBP7 promoted apoptosis as evidenced by enhanced cell viability and suppressed cell apoptosis in OA chondrocytes after miR-25-3p overexpression or IGFBP7 knockdown. The miR-25-3p facilitated chondrocyte viability and repressed cell apoptosis in OA by negatively regulating IGFBP7.

Conclusions. MiR-25-3p negatively regulates IGFBP7 to promote chondrocyte proliferation and restrain chondrocyte apoptosis. Our findings suggest that the regulation of IGFBP7 by miR-25-3p may emerge as a novel therapeutic regimen for OA. (Folia Histochemica et Cytobiologica 2021, Vol. 59, No. 1, 30-39)
\end{abstract}

Key words: miR-25-3p; insulin-like growth factor-binding protein 7; osteoarthritis; chondrocyte; proliferation; apoptosis

\section{Introduction}

Osteoarthritis $(\mathrm{OA})$ has been defined as a chronic joint malady featured by degeneration of ligaments and articular cartilage, thickening of subchondral bone and generation of osteophytes [1,2]. The prevalence of OA worldwide is staggeringly high with an estimated over 250 million people affected and with a lifetime risk for worsening to knee OA of approxi-

Correspondence address: Xiao $\mathrm{He}$

The Joint Surgical Center,

Chenzhou No. 1 People's Hospital, No. 102, Luojiajin,

Beihu District, Chenzhou, Hunan 423000, China

phone: +86-15973507312, e-mail: hexiao8211@163.com mately $40 \%$ [3]. Chondrocyte, a primary cell type of articular cartilage which occupies approximately $1 \%$ of total cartilage volume, is essential for retaining the dynamic equilibrium between catabolism and anabolism of the extracellular matrix in articular cartilage and has been deemed as one of the indispensable regulators of OA pathogenesis $[4,5]$. Declining cell number and cell viability of chondrocytes is implicated in the progression and advancement of OA [6]. Thus, the prevention of chondrocyte apoptosis and the promotion of chondrocyte proliferation may be potential therapeutic strategies for OA treatment.

MicroRNAs (miRNAs) play roles in a variety of physiological functions and disease processes by mediating cleavage and destabilization of mRNA [7].

This article is available in open access under Creative Common Attribution-Non-Commercial-No Derivatives 4.0 International (CC BY-NC-ND 4.0) license, allowing to download articles and share them with others as long as they credit the authors and the publisher, but without permission to change them in any way or use them commercially. (c) Polish Society for Histochemistry and Cytochemistry 
Zhixi Duan et al. have illustrated that miR-15a-5p motivates the degeneration and proliferation of chondrocytes through PTHrP inhibition [8]. Another study describes that the promotion of chondrocyte apoptosis and suppression of chondrocyte proliferation in OA can be caused by miR-181 [9]. These studies emphasize the possibility that miRNAs are associated with OA pathogenesis. In neurons, miR-25-3p was shown to suppress epileptiform discharges by hampering apoptosis and oxidative stress [10]. Besides, miR-25 possesses an inhibitory effect on sepsis-induced cardiomyocyte apoptosis [11]. However, the definite mechanism of miR-25-3p in the apoptosis of chondrocytes remains largely unexplored. Serving as a low-affinity insulin growth factor binder, insulin-like growth factor-binding protein 7 (IGFBP7) may confer significant protective effects on bone metabolism [12]. In breast cancer, IGFBP7 can block cancer cell growth through induction of apoptosis and senescence [13]. Additionally, the elevated IGFBP7 expression in human OA chondrocytes has been recently reported [14]. Accordingly, IGFBP7 may represent an up-and-coming target during the therapy of apoptosis-related OA. Herein, this study presented that miR-25-3p was significantly suppressed and IGFBP7 was remarkably elevated in TNF- $\alpha$-induced rat chondrocytes. Overexpression of miR-25-3p significantly enhanced cell viability and inhibited cell apoptosis in rat chondrocytes in vitro. Interestingly, we corroborated that miR-25-3p regulates IGFBP7 by directly targeting the 3'-UTR of IGFBP7. Our findings suggest that the regulation of IGFBP7 by miR-25-3p may emerge as a novel therapeutic regimen for $\mathrm{OA}$.

\section{Materials and methods}

Ethical statement. The experimental scheme was authorized by the Research Ethics Committee of Chenzhou No. 1 People's Hospital. All procedures involving animals were in compliance with the Guide for the Care and Use of Laboratory Animals.

Cell culture. Male Wistar rats ( $140 \pm 10 \mathrm{~g})$ were supplied by Laboratory Animal Center, Chinese Academy of Sciences (Beijing, China). Rats were anesthetized and sacrificed by cervical dislocation. Then chondrocytes were isolated from cartilage tissues of rats under sterile conditions. In brief, cartilage was digested with trypsin (Thermo, Waltham, MA, USA) at $37^{\circ} \mathrm{C}$ for $30 \mathrm{~min}$ and then with $0.2 \%$ type II collagenase (Thermo) for $3 \mathrm{~h}$ at $37^{\circ} \mathrm{C}$. Five min of shaking every $1 \mathrm{~h}$ in a constant temperature container was necessary until the fragments were digested. When cartilage mass was visible to the naked eye in floccule shape, isolated chondrocytes were observed under an inverted microscope. Cell digestion was terminated by exposure to fetal bovine serum
(FBS, Gibco, Carlsbad, CA) and stroking with a pipette tip. After that, cells were filtrated (200 mesh), centrifuged $(1,500 \mathrm{rpm} \times 10 \mathrm{~min})$ and washed $3 \times$ with phosphate-buffered saline (PBS). Cells were cultured with Dulbecco's modified Eagle medium (DMEM) containing 10\% of FBS and $1 \%$ of penicillin/streptomycin (Solarbio, Beijing, China) in an incubator (Thermo) at $37^{\circ} \mathrm{C}$ in $5 \% \mathrm{CO}_{2}$. After $48 \mathrm{~h}$ of incubation, the culture medium was changed to remove the non-adherent cells, followed by replacement every other day. Cell morphology and adherent growth were recorded under an inverted microscope. The passage was conducted after the cell adherence to $85 \% \sim 90 \%$. The chondrocytes of the $3^{\text {rd }}$ to $5^{\text {th }}$ generation were utilized in the experiments. Identification of chondrocytes was performed using toluidine blue staining and immunofluorescent staining of type II collagenase.

Toluidine blue staining [15]. The adherent chondrocytes were digested to prepare a cell suspension, and then cells $\left(1 \times 10^{5} / \mathrm{mL}\right)$ were seeded into six-well plates for incubation. When cells were completely attached and the cell confluence reached approximately $80 \%$, the culture medium was discarded before the cells were washed $3 \times$ in PBS. Cells in each well were fixed in $4 \%$ paraformaldehyde $(2 \mathrm{~mL})$ in refrigerator at $4^{\circ} \mathrm{C}$ for $1 \mathrm{~h}$, followed by three times of PBS washing, exposure to $1 \mathrm{~mL}$ of toluidine blue (an appropriate stain for detecting aggrecan, Sangon Biotech, Shanghai, China) at room temperature for $1 \mathrm{~h}$, and toluidine blue was removed after washing $3 \times$ with PBS. The inverted microscope was utilized for observation of the cells.

Immunofluorescent staining. Cells $\left(1 \times 10^{5} / \mathrm{mL}\right)$ were placed on disposable confocal dishes for incubation, prior to three times of PBS rinsing, $30 \mathrm{~min}$ of fixation in $2 \mathrm{~mL}$ of $4 \%$ paraformaldehyde at room temperature, $3 \times$ of PBS rinsing, 20 min of permeation with $1 \mathrm{~mL}$ of $0.1 \%$ TritonX-100 solution at room temperature and $3 \times$ of PBS rinsing. After that, cells were blocked with $1 \mathrm{~mL}$ of $10 \%$ goat serum (Zsbio, Beijing, China) at room temperature for $30 \mathrm{~min}$. Cells were incubated with primary antibody $(1 \mathrm{~mL})$ against $10 \%$ goat serum-diluted rabbit anti-type II collagen (ab34712, 1:200, Abcam, Cambridge, MA, USA) overnight in the dark at $4^{\circ} \mathrm{C}$, and then washed $3 \times$ with PBS. Following incubation with the secondary antibody $(1 \mathrm{~mL})$ against $10 \%$ goat serum-diluted Alexa Fluor (ab150077, 1:500, Abcam) at $37^{\circ} \mathrm{C}$ for $1 \mathrm{~h}$ and washing $3 \times$ with PBS, cells were immersed in $1 \mathrm{~mL}$ of DAPI (SigmaAldrich, Merck KGaA, Darmstadt, Germany) at room temperature for $2 \mathrm{~min}$, followed by washing $3 \times$ with PBS. Images were captured by a laser scanning confocal microscopy $(200 \times$, green light: wavelength of $543 \mathrm{~nm}$, blue light: wavelength of $458 \mathrm{~nm}$, Olympus OLS5000, Tokyo, Japan) after the cells were covered with $1 \mathrm{~mL}$ of anti-fading mounting medium.

Establishment of OA cellular model. TNF- $\alpha$ is a commonly used stimulus to establish OA cellular models [16]. In the present study, $20 \mathrm{ng} / \mathrm{mL}$ of TNF- $\alpha$ (PeproTech, Rocky Hill, 
Table 1. Primers' sequences

\begin{tabular}{|l|l|l|}
\hline Name & & Sequences $\left(\mathbf{5}^{\prime}-\mathbf{3}\right.$ ') \\
\hline \multirow{2}{*}{ miR-25-3p } & Forward & CATTGCACTTGTCTCGGTCTGA \\
\cline { 2 - 3 } & Reverse & GCTGTCAACGATACGCTACGTAACG \\
\hline \multirow{2}{*}{ IGFBP7 } & Forward & UUCUCCGAACGUGUCACGUTT \\
\cline { 2 - 3 } & Reverse & UGACACGUUCGGAGAATT \\
\hline \multirow{2}{*}{ GAPDH } & Forward & CGAGCAAGGTCCTTCCATAGT \\
\cline { 2 - 3 } & Reverse & GGTGTCGGGATTCCGATGAC \\
\hline & Forward & GGAGCGAGATCCCTCCAAAAT \\
\cline { 2 - 3 } & Reverse & GGCTGTTGTCATACTTCTCATGG \\
\hline
\end{tabular}

NJ, USA) was used to stimulate chondrocytes. Briefly, cells were immersed in culture medium with $20 \mathrm{ng} / \mathrm{mL}$ of TNF- $\alpha$ for $6 \mathrm{~h}$, and then the medium was changed into normal culture medium for $24 \mathrm{~h}$ of additional culture. Cells in the OA group were treated with $20 \mathrm{ng} / \mathrm{mL}$ of TNF- $\alpha$ for $6 \mathrm{~h}$ of OA modeling, and cells in the Sham group were cultured in normal medium. To determine expression levels of the studied molecules and functional tests, cells were seeded onto six-well plates and exposed to the Lipofectamine 2000 reagent for cell transfection. After that, cells were cultured in normal medium for $24 \mathrm{~h}$ prior to $6 \mathrm{~h}$ of $20 \mathrm{ng} / \mathrm{mL} \mathrm{TNF}-\alpha$ treatment, and then the medium containing TNF- $\alpha$ was replaced with normal medium for $24 \mathrm{~h}$ of additional incubation. Then the levels of miR-25-3p and IGFBP7 were measured as described below.

Cell transfection and grouping. The miR-25-3p mimic, mimic-NC (100 nM), miR-25-3p inhibitor, inhibitor-NC (100 nM), pcDNA3.1-IGFBP7 and pcDNA3.1 $(2 \mu \mathrm{g})$ were supplied by GenePharma (Shanghai, China). When cell confluence reached $70 \% \sim 80 \%$, cell transfection was performed according to the introduction provided with the Lipofectamine 2000 kit (Invitrogen, Carlsbad, CA, USA). Cells were transfected with above plasmids and accordingly grouped into the miR-25-3p mimic group, mimic-NC group, miR-25-3p inhibitor group, inhibitor-NC group, pcDNA3.1-IGFBP7 group, pcDNA3.1 group and Blank group.

Quantitative reverse transcription polymerase chain reaction (qRT-PCR). Total RNA was obtained from chondrocytes by utilizing TRIzol (Invitrogen). Reverse transcription was performed with the reverse transcription kit (TaKaRa, Tokyo, Japan). All operations were conducted based on the manufacturer's instructions. The expression of gene was inspected by LightCycler 480 qPCR instrument (Roche, Indianapolis, IN, USA), and reaction condition was instructed by the fluorescence quantitative PCR kit (SYBR Green Mix, Roche Diagnostics). The real-time PCR program was as follows: $95^{\circ} \mathrm{C}$ for $10 \mathrm{~s}$, followed by 45 cycles of $95^{\circ} \mathrm{C}$ for $5 \mathrm{~s}, 60^{\circ} \mathrm{C}$ for $10 \mathrm{~s}$ and $72^{\circ} \mathrm{C}$ for $10 \mathrm{~s}$.
A final extension was performed at $72^{\circ} \mathrm{C}$ for $5 \mathrm{~min}$. The experiments were done in triplicate. The internal reference was GAPDH and data analysis utilized $2^{-\Delta \Delta \mathrm{Ct}}$ method. The formula is as follows: $\Delta \Delta \mathrm{Ct}=\left[\mathrm{Ct}_{\text {(target gene) }}-\mathrm{Ct}_{\text {(reference gene) }}\right]$ experimental group $-\left[\mathrm{Ct}_{\text {(target gene) }}-\mathrm{Ct}_{\text {(reference gene) }}\right]_{\text {control group }}$. The primer sequences are presented in Table 1.

Western blotting. Cells were lysed with RIPA lysate (Beyotime Biotechnology, Shanghai, China) to obtain protein samples. After the protein concentration was analyzed by a BCA kit (Beyotime), the corresponding volume of protein was added to the loading buffer (Beyotime) and mixed. The solution containing proteins was placed in a boiling-water bath for $5 \mathrm{~min}$ of denaturation. Electrophoresis was conducted for $30 \mathrm{~min}$ at $80 \mathrm{~V}$ and then for $1 \sim 2 \mathrm{~h}$ at $120 \mathrm{~V}$ once bromphenol blue reached the separation gel. Then, the proteins were transferred onto membranes at $220 \mathrm{~mA}$ for $120 \mathrm{~min}$ in an ice-bath. The membranes were rinsed $1 \sim 2$ min with washing solution and sealed in the blocking solution at room temperature for $60 \mathrm{~min}$. After blocking, the membranes were incubated with the primary antibodies against GAPDH (5174S, 1:1000, Cell Signaling, Boston, USA), IGFBP7 (ab74169, 1:1000, Abcam) and cleaved caspase-3 (ab49822, 1:1000, Abcam) overnight at $4^{\circ} \mathrm{C}$ on a shaking table. Following incubation, the membranes were washed $3 \times$ with washing solution for $10 \mathrm{~min}$ before incubation with horseradish peroxidase-labeled goat anti-rabbit $\operatorname{IgG}$ (1:5000, Beijing ComWin Biotech Co., Ltd., Beijing, China) for $1 \mathrm{~h}$ at room temperature. The membranes were washed $3 \mathrm{x}$ for $10 \mathrm{~min}$ and exposed to developing liquid for color development. Then chemiluminescence imaging analysis system (Bio-rad, Hercules, CA, USA) was utilized for observation.

Flow cytometry. One milliliter of cell suspension was seeded onto a six-well plate $\left(5 \times 10^{5}\right.$ cells/well $)$ and cultured in an incubator at $37^{\circ} \mathrm{C}$ in $5 \% \mathrm{CO}_{2}$. After $24 \mathrm{~h}$, adherent cells were digested by trypsin, washed with precooled PBS twice $(2,000 \mathrm{rpm}$, $5 \mathrm{~min}$ ) and re-suspended with $1 \mathrm{~mL}$ of Annexin $\mathrm{V}$ binding buffer. Flow cytometry (flow cytometer, FACSCalibur, BD Biosciences) was utilized to assess cell apoptosis after 
cell exposure to $10 \mu \mathrm{L}$ of propidium iodide (PI) and $5 \mu \mathrm{L}$ of Annexin V-FITC for $30 \mathrm{~min}$ at room temperature in the dark. The experiments were done in triplicate.

CCK-8 assay. The cell viability was measured with CCK-8 assay kit (CCK-8, Merck KGaA). One hundred microliters of cell suspension in each well of 96-well plates (1,500 cells /well) was incubated at $37^{\circ} \mathrm{C}$ in $5 \% \mathrm{CO}_{2}$. After $24 \mathrm{~h}$ of pre-incubation, $10 \mu \mathrm{L}$ of CCK- 8 was added for $2 \mathrm{~h}$ of additional incubation. The absorbance (optical density, OD) at the wavelength of $450 \mathrm{~nm}$ was determined by using a microplate reader, and the experiment was repeated $3 \times$. The absorbance value $\mathrm{A}$ was measured and the proliferation rate was reckoned. Proliferation rate $(\%)=\left[\mathrm{A}_{\text {(transfection group) }}-\mathrm{A}_{(\text {Control) }}\right] /$ $/\left[\mathrm{A}_{\text {(Blank group) }}-\mathrm{A}_{\text {(Control) }}\right] \times 100 \% \mathrm{~A}_{\text {(transfection group) }}$ : absorbance value of cells subjected to cell transfection and CCK-8 solution; $\mathrm{A}_{\text {(Control) }}$ : absorbance value of cells incubated with culture medium and CCK-8 solution; $\mathrm{A}_{\text {(Blank group) }}$ : absorbance value of cells without cell transfection but incubated with CCK-8 solution.

Dual-luciferase reporter gene assay. The TargetScan (http:// www.targetscan.org/vert_72/) was used to predict the binding site of miR-25-3p and IGFBP7. The mutated and wild type sequences in the binding site of miR-25-3p and IGFBP7 were determined in accordance with the predicted results (mut-IGFBP7, wt-IGFBP7) and cloned into luciferase expression vectors (pGL3-Basic). Then the vectors were cotransfected with miR-25-5p (0, $50 \mathrm{nM}, 300 \mathrm{nM}$, Gene Pharma, Shanghai, China) into HEK293T cells. After cell transfection, HEK293T cells were incubated with $100 \mu \mathrm{L}$ of cell lysis buffer on a shaking table at room temperature for $20 \mathrm{~min}$ to lyse the cells. Firefly luciferase activity or Renilla luciferase activity was measured after cell suspension exposure to $50 \mu \mathrm{L}$ of luciferase reaction solution (Promega, Madison, WI, USA) or $50 \mu \mathrm{L}$ of Stop\&Glo reagent (Promega). The relative activity was calculated as the ratio of firefly luciferase activity to Renilla luciferase activity. Renilla luciferase activity was regarded as the internal control. Three replicates were set for this test.

Statistical analysis. Statistical analysis was conducted by utilizing GraphPad Prism 5 software (GraphPad Software Inc., San Diego, CA, USA). Student's $t$-test was adopted for the comparison between two groups. Dunnett's multiple comparisons test for comparisons among multiple groups, and the Pearson correlation coefficient was applied to assess the correlation among parameters. $P$ values of significance were at $p<0.05$.

\section{Results}

\section{Identification of rat chondrocytes and $\mathrm{OA}$ cellular model}

The morphology of isolated rat chondrocytes was identified by an inverted microscopy. As depicted in Figure 1A, the rat chondrocytes are grown adherent to the wall, arranged in an irregularly rounded or polygonal pattern and covered the bottom of dishes. Toluidine blue staining of aggrecan secreted by rat chondrocytes exhibited that the cell membrane and cytoplasm of chondrocytes were in blue-purple and the nuclei were in dark purple with obvious nucleoli (Fig. 1B). Results of immunofluorescent staining of specific type II collagenase secreted by rat chondrocytes expounded that the addition of specific type II collagen antibodies stained the cytoplasm to green, and DAPI stained nucleus to blue (Fig. 1C). These data indicated that the cells isolated from rat knee joints are chondrocytes.

After TNF- $\alpha$ stimulation for OA modeling, CCK- 8 assay, flow cytometry and Western blotting were adopted to evaluate the model establishment. These results manifested that the OA group had suppressed proliferation rate (Fig. 1D, $p<0.01$ ), elevated apoptosis rate (Fig. 1E, $p<0.01$ ) and enhanced cleaved caspase-3 expression (Fig. 1F, $p<0.01$ ) when compared with the Sham group. The above results proved that the cells isolated and extracted in this experiment are primary rat chondrocytes from the knee joint, and the apoptosis of chondrocytes could be induced by TNF- $\alpha$. The successfully established OA cellular models could be used for subsequent experiments.

\section{miR-25-3p facilitates cell proliferation and represses apoptosis in TNF- $\alpha$-induced chondrocytes}

To ascertain the role of miR-25-3p in cell apoptosis and proliferation under the OA-like condition, rat chondrocytes were transfected with miR-25-3p mimic, miR-25-3p inhibitor as well as corresponding negative controls. qRT-PCR described that OA modeling decreased the expression of miR-25-3p in rat chondrocytes (Fig. 2A, $p<0.05$, vs. sham group). Transfection with miR-25-3p mimic heightened miR-25-3p level (Fig. 2B, $p<0.05$, vs. mimic-NC group), while transfection with miR-25-3p inhibitor inhibited miR-25-3p expression in the rat chondrocytes (Fig. $2 \mathrm{~B}, p<0.05$, $v s$. inhibitor-NC group), suggesting that miR-25-3p mimic and inhibitor have good transfection efficiencies and can be used in the subsequent experiments.

CCK-8 assay and flow cytometry were performed to further identify the effect of miR-25-3p on the activity of TNF- $\alpha$-induced rat chondrocytes, and results revealed that the OA + miR-25-3p mimic group possessed elevated cell proliferation rate (Fig. $2 \mathrm{C}, p<0.05$ ) and repressed cell apoptosis (Fig. 2D, $p<0.05$ ) in comparison to the $\mathrm{OA}+$ mimic $\mathrm{NC}$ group, while the fall in cell proliferation (Fig. $2 \mathrm{C}, p<0.05$ ) and the rise in cell apoptosis (Fig. 2D, $p<0.05$ ) were observed in OA + miR-25-3p inhibitor group when compared with the $\mathrm{OA}+$ inhibitor NC group. No significant 


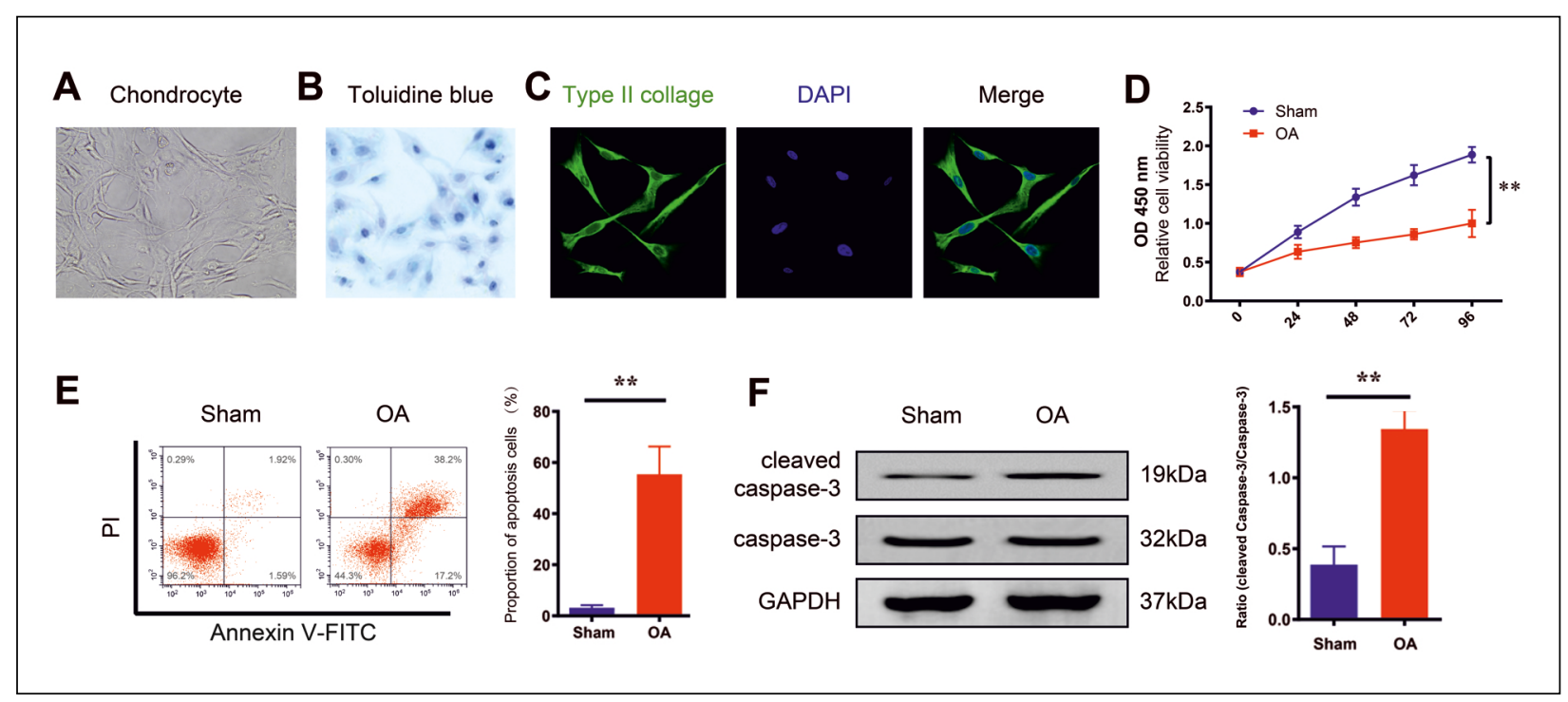

Figure 1. Isolation of rat chondrocytes and establishment of osteoarthritis (OA) cellular model. A. The morphology of rat chondrocytes was observed by inverted microscopy, Scale bar indicates $100 \mu \mathrm{m}$. B. Toluidine blue staining (purple, $100 \times$ ). C. Type II collagen staining (green, 200×), and DAPI staining (blue, 200×). The cell proliferation rate was determined by CCK-8 assay (D), cell apoptosis rate was assessed by flow cytometry (E) and protein level of cleaved caspase-3 was measured by Western blotting $(\mathbf{F}) .{ }^{* *} p<0.01$.

differences among the OA group, $\mathrm{OA}+$ mimic $\mathrm{NC}$ group and $\mathrm{OA}+$ inhibitor $\mathrm{NC}$ group were noted with regard to those indices $(p>0.05)$.

Western blotting showed that the OA + miR-25-3p mimic group had decreased cleaved caspase- 3 protein expression (Fig. $2 \mathrm{E}, p<0.05$, $v$ s. OA + mimic $\mathrm{NC}$ group), whereas the OA + miR-25-3p inhibitor group had increased cleaved caspase-3 (Fig. 2E, $p<0.05$, vs. OA + inhibitor NC group). These findings illustrated that miR-25-3p can enhance viability of rat chondrocytes and suppress apoptosis of TNF$-\alpha$-induced chondrocytes.

\section{TNF- $\alpha$-induced chondrocyte apoptosis is promoted by IGFBP7}

This study was undertaken to explore the role of IGFBP7 in OA. Analyses of qRT-PCR and Western blotting manifested that the mRNA and protein levels of IGFBP7 were increased in rat chondrocytes following TNF- $\alpha$ induction (Fig. 3A, $p<0.05$, $v s$. sham group). Additionally, overexpression of IGFBP7 heightened IGFBP7 expression (Fig. 3B, $p<0.05$, vs. pcDNA3.1 group), while knockdown of IGFBP7 diminished IGFBP7 expression (Fig. 3B, $p<0.05$, vs. si-NC group). These results indicated good transfection efficiencies of si-IGFBP7 and pcDNA3.1-IGFBP7.

Results of CCK-8 assay, flow cytometry and Western blotting revealed that transfection with pcDNA3.1-IGFBP7 elevated cell apoptosis (Fig. 3D, $p<0.05$, vs. OA + pcDNA3.1 group) and protein level of cleaved caspase-3 (Fig. 3E, $p<0.05$ ), along with decreased cell viability (Fig. 3C, $p<0.05$ ) of rat chondrocytes, while transfection with si-IGFBP7 had opposite findings (Fig. 3C-E, $p<0.05$, OA + si-IGFBP7 group $v s$. OA + si-NC group). There were no remarkable differences in these factors among the $\mathrm{OA}, \mathrm{OA}+$ si-NC and OA + pcDNA3.1 groups. Collectively, IGFBP7 can accelerate TNF- $\alpha$-induced chondrocyte apoptosis and inhibit cell proliferation.

\section{miR-25-3p negatively mediates IGFBP7}

The aforementioned results have addressed that miR-25-3p and IGFBP7 play opposite roles in the regulation of chondrocyte viability and apoptosis. Subsequently, whether miR-25-3p can regulate IGFBP7 in chondrocytes needs to be investigated. Prediction by the online biological software StarBase 2.0 displayed that miR-25-3p had a binding site with IGFBP7. Dual-luciferase reporter gene assay exhibited that the luciferase activity of HEK293T cells in the IGFBP7 3'-UTR WT group was decreased by transfection of miR-25-3p mimic in a dose dependent manner (Fig. 4A, $p<0.05$ ). While transfection with miR-25-3p mimic had no significant impact on the luciferase activity of HEK293T cells in the IGFBP7 3'-UTR MUT group, indicating the binding of miR25-3p and IGFBP7. qRT-PCR and Western blotting further corroborated this finding. Transfection with miR-25-3p mimic suppressed IGFBP7 in rat chon- 


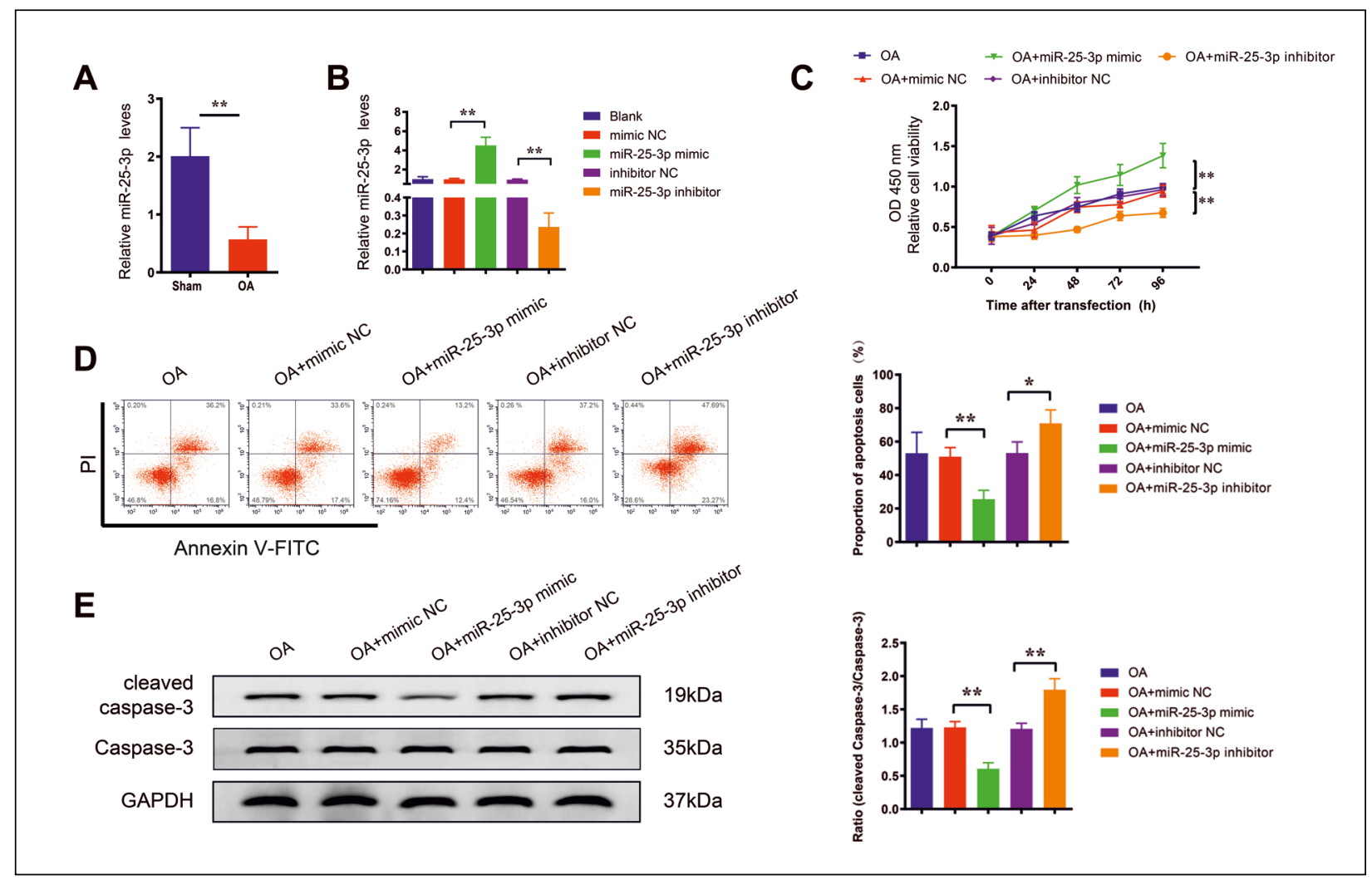

Figure 2. MiR-25-3p enhances the viability and reduces the apoptosis of TNF- $\alpha$-induced rat chondrocytes. qRT-PCR detection of miR-25-3p expression in rat chondrocytes after OA modeling (A) and the transfection efficiency of miR-25-3p mimic and inhibitor (B). CCK-8 assay was used to measure the effect of miR-25-3p on chondrocyte viability (C). The role of miR-25-3p in cell apoptosis after OA modeling was determined by flow cytometry (D). Western blotting was utilized to detect the effect of miR-25-3p on protein expression of cleaved caspase-3 after TNF- $\alpha$ induction (E). ${ }^{*} p<0.05,{ }^{* *} p<0.01$, $\mathrm{OA}$ - osteoarthritis.

drocytes (Fig. 4B-C, p $<0.05$, miR-25-3p mimic group vs. mimic $\mathrm{NC}$ group), whereas transfection with miR-25-3p inhibitor raised IGFBP7 level (Fig. 4B-C, $p<0.05$, miR-25-3p inhibitor group $v s$. inhibitor NC group). Taken together, miR-25-3p may negatively regulate IGFBP7.

\section{miR-25-3p negatively targets IGFBP7 to inhibit the apoptosis of $T N F-\alpha$-induced rat chondrocytes} It will be further verified whether miR-25-3p could exert its effect on chondrocyte apoptosis by regulating IGFBP7. CCK-8 assay, flow cytometry and Western blotting uncovered that there were increased cell viability (Fig. 5A, $p<0.05$ ), repressed cell apoptosis (Fig. 5B, $p<0.05$ ) and decreased cleaved caspase-3 expression (Fig. 5C, $p<0.05$ ) in the OA + miR-25-3p mimic group rather than in the OA group or OA + miR-25-3p mimic + pcDNA3.1-IGFBP7 group. Furthermore, overexpression of IGFBP7 in OA chondrocytes heightened cell apoptosis rate, increased cleaved caspase- 3 expression, and diminished cell viability (Fig. 5A-C, $p<0.05$, OA group $v s$. OA +
pcDNA3.1-IGFBP7 group), while following exposure to miR-25-3p mimic revered these trends (Fig. 5A-C, $p<0.05, \mathrm{OA}+$ pcDNA3.1-IGFBP7 group vs. OA + miR-25-3p mimic + pcDNA3.1-IGFBP7 group). These data implicated that miR-25-3p may negatively target IGFBP7 to promote proliferation and restrain apoptosis of rat chondrocytes induced by TNF- $\alpha$.

\section{Discussion}

OA, typified by joint space narrowing and a degenerative loss of cartilage integrity, is a dominant reason for disability, pain, and shortening of adult working life [17]. Interestingly, there is evidence showing that apoptosis is progressively recognized as a crucial driver of OA cartilage pathology $[18,19]$. Dysregulated gene expression in chondrocytes is implicated in the apoptosis and proliferation of chondrocytes, emphasizing the functional role of miRNAs in controlling chondrocyte apoptosis and development of OA [9, 20]. In the present study, the chondrocytes were isolated from cartilage tissues of rats under sterile con- 


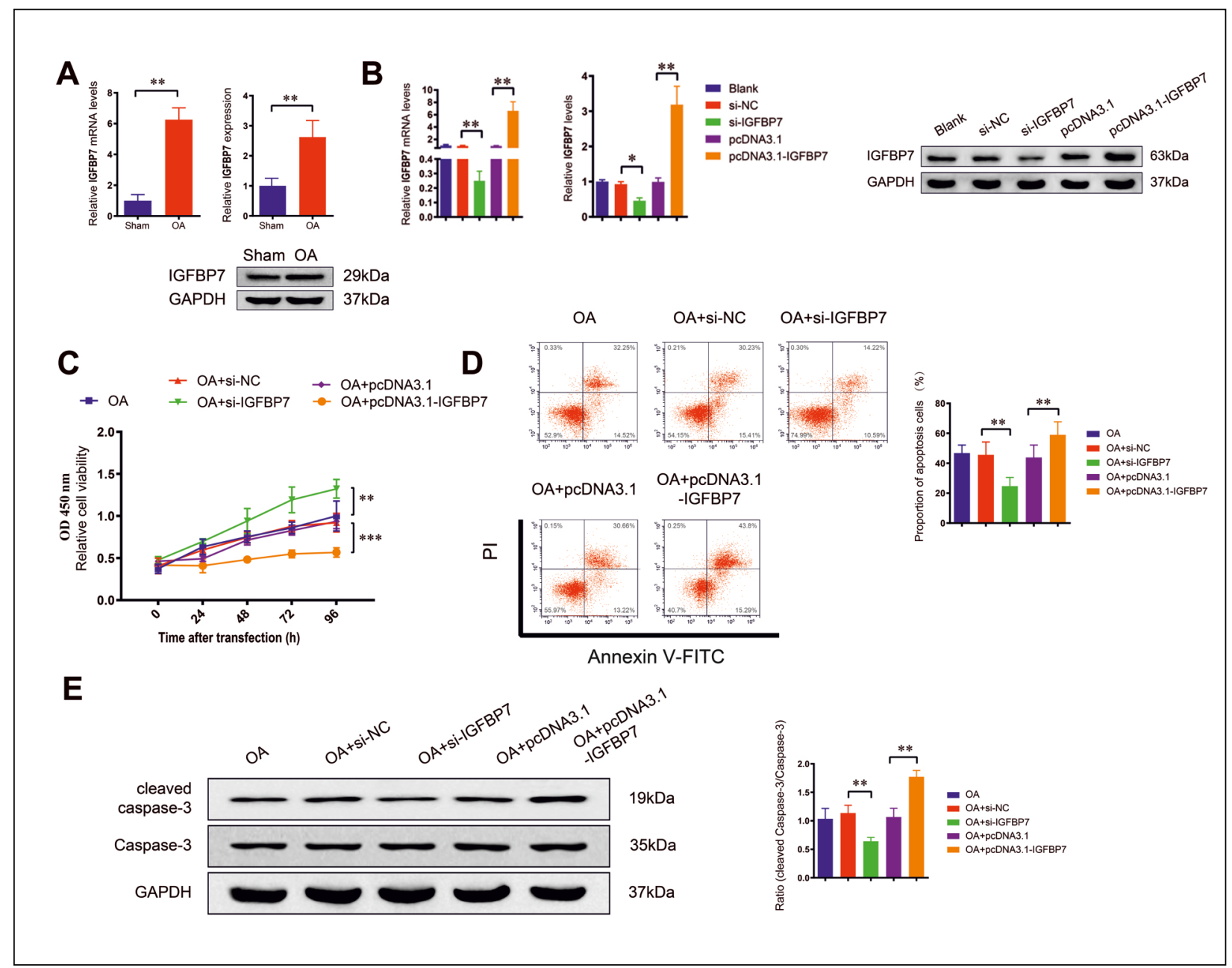

Figure 3. IGFBP7 pushes the apoptosis of TNF- $\alpha$-induced rat chondrocytes. A. The IGFBP7 expression in chondrocytes after OA modeling was determined by qRT-PCR and Western blotting. B. The transfection efficiencies of si-IGFBP7 and pcDNA3.1-IGFBP7 were measured by qRT-PCR. CCK-8 assay was used for the assessment of chondrocyte viability (C), flow cytometry for determination of cell apoptosis after OA modeling (D), and Western blotting for measurement of cleaved caspase-3 protein expression after TNF- $\alpha$ induction (E). ${ }^{*} p<0.05,{ }^{* *} p<0.01,{ }^{* * *} p<0.001$; OA - osteoarthritis.

ditions, cell cultures were established and then cells were induced with TNF- $\alpha$. Subsequently, the effects of miR-25-3p on the proliferation and apoptosis of chondrocytes were researched in this study to reveal OA etiology. The results from our study suggested that miR-25-3p represses the apoptosis and promotes the proliferation of rat chondrocytes under the OAlike condition by IGFBP7 suppression.

Initially, we identified that the morphology of isolated cells shared some characteristics with chondrocytes. Furthermore, these findings were further supported by the results of toluidine blue staining of aggrecan and immunofluorescent staining of specific type II collagenase, which suggested that the isolated cells from rats are chondrocytes. Then, the chondrocytes were exposed to $20 \mathrm{ng} / \mathrm{mL}$ of TNF- $\alpha$ to induce OA in chondrocytes. A series of analyses demonstrated that TNF- $\alpha$ induction caused apoptosis of chondrocytes, indicating successful OA cellular model. The expression of miR-25-3p was significantly decreased in the in vitro OA model. This has been previously suggested by a bioinformatical analysis of the osteoarthritis-associated miRNA [21-25]. Additionally, miR-25-3p was found to interfere with the proliferation and apoptosis of chondrocytes under the OA-like condition. As a member of the miR-106b-25 cluster, miR-25 has been identified to be abnormally expressed in various types of tumor and to confer functional roles during a variety of tumor-related processes, including tumorigenesis and cancer cell migration, proliferation and metastasis [26]. For example, miR-25-3p functions as an oncogenic miRNA in osteosarcoma by targeting 
A

WT IGFBP7 3'-UTR $\quad$..CACUCAUCAUUCGCGGUGCAAUA...
has-miR-25-3p
MUG IGFBP7 3'-UTR
...CACUCAUCAUUCGCGACUACAGA...

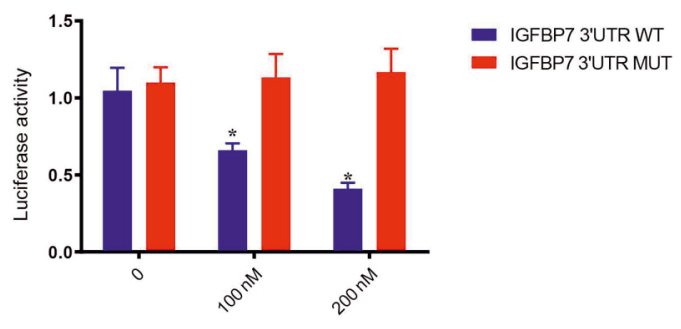

C

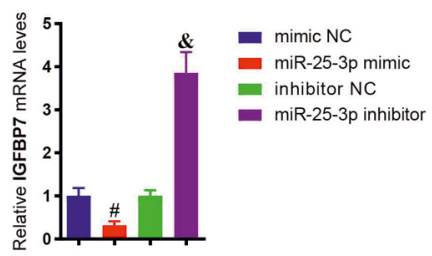

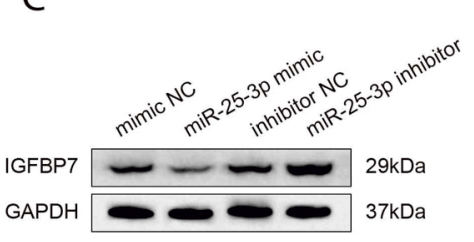

- mimic $\mathrm{NC}$

- inhibitor NC

- miR-25-3p inhibitor
miR-25-3p mimic

GAPDH
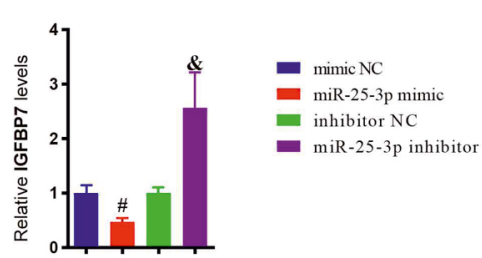

Figure 4. IGFBP7 is negatively regulated by miR-25-3p in rat chondrocytes. A. Left: the binding site and mutation site of IGFBP7 and miR-25-3p; right: the binding of miR-25-3p to IGFBP7 displayed by dual-luciferase reporter gene assay. The regulation of miR-25-3p on the mRNA and protein levels of IGFBP7 was exhibited by qRT-PCR (B) and Western blotting (C). ${ }^{*} p<0.05$ compared to IGFBP7 3'UTR WT group; ${ }^{*} p<0.05$ compared to mimic NC group. ${ }^{*} p<0.05$ compared to inhibitor NC group. WT — wild type; NC — negative control; UTR — untranslated region.

A

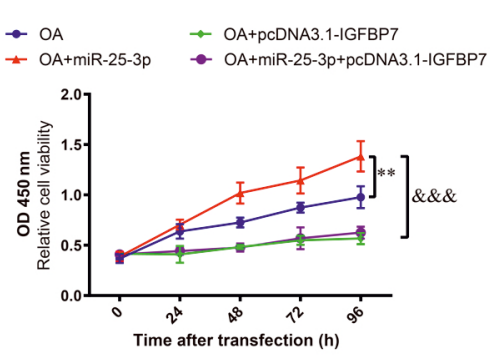

C

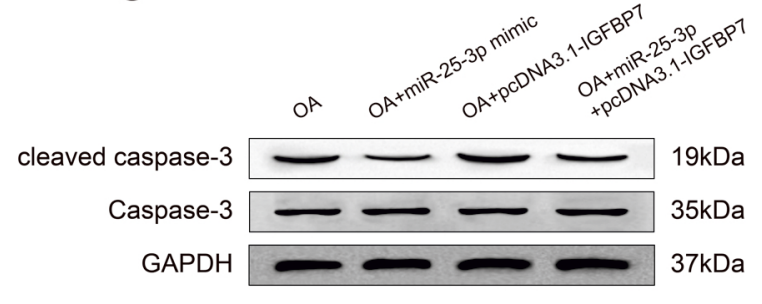

B

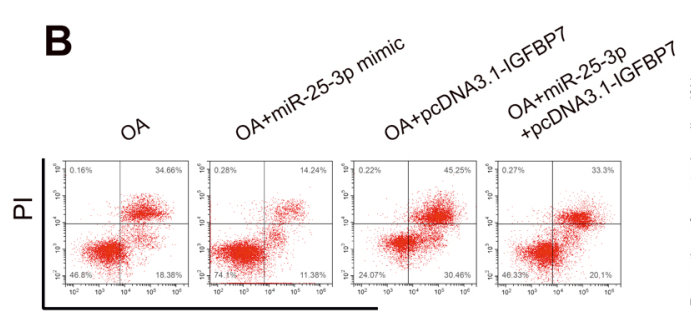

Annexin V-FITC
OA

OA+miR-25-3p

OA+miR-25-3p+PCDNA3.1-IGFBP7

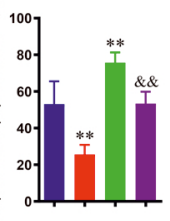

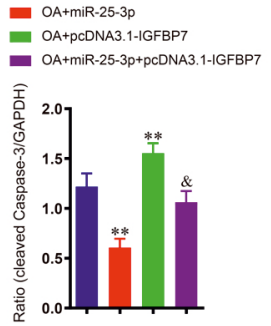

Figure 5. MiR-25-3p inhibits the apoptosis of TNF- $\alpha$-induced rat chondrocytes by suppressing IGFBP7. CCK-8 assay was utilized for inspection of chondrocyte proliferation rate (A), flow cytometry for detection of cell apoptosis rate (B) and Western blotting for assessment of cleaved caspase-3 expression (C). ${ }^{* *} p<0.01$ compared to OA group; ${ }^{\star} p<0.05$, \&\& $p<0.01,{ }^{\& \&} p<0.001$ compared to OA + miR-25-3p group; OA - osteoarthritis.

Merlin, and may serve as a potential therapeutic target for osteosarcoma [27]. Previously, miR-25 was expounded to involve in the metastasis and proliferation of non-small cell lung cancer by targeting the LATS2/ /YAP pathway [28]. Moreover, in triple-negative breast cancer, the biological effects of miR-25-3p on cell apoptosis and proliferation through BTG2 mediation and subsequent activation of AKT and ERKMAPK pathway were also elucidated in a recent study [29]. In agreement with recent studies, we found that 
miR-25-3p facilitated proliferation and suppressed apoptosis of TNF- $\alpha$-induced chondrocytes. Our finding was subsequently corroborated by the loss and gain of miR-25-3p function, which addressed that overexpression of miR-25-3p enhanced cell viability, decreased cell apoptosis and diminished cleaved caspase-3, while knockdown of miR-25-3p promoted cell apoptosis and repressed cell proliferation.

The second novel observation of this study that has not been reported before is the finding of a possible role of IGFBP7 in the pathomechanisms of OA because we showed that there is a reciprocal relationship between the expression of miR-25-3p and IGFBP7 in the TNF- $\alpha$-induced OA model. In the current study, the expression level of IGFBP7 was found to be increased in rat chondrocytes following TNF- $\alpha$ induction. IGFBP7 is a protein secreted from the IGFBP family [30]. In this study, IGFBP7 conferred crucial effects on the biological behaviors of TNF- $\alpha$-induced rat chondrocytes. A lot of experiments illustrated that IGFBP7 facilitated TNF- $\alpha$-induced chondrocyte apoptosis and inhibited cell proliferation. The previous study has illustrated that IGFBP7 restrains thyroid carcinoma cell proliferation through suppressing cell cycle progression and AKT activity [31]. IGFBP7 is reported to trigger the apoptosis of acute myeloid leukemia cell and to synergize with chemotherapy in suppressing leukemia cells [32]. Additionally, IGFBP7 may exert a therapeutic role in estrogen deficiency-induced osteoporosis [12]. With regard to these evidences, we ensured that IGFBP7 plays an indispensable role in the proliferation and apoptosis of TNF- $\alpha$-induced rat chondrocytes. Subsequently, the online biological software StarBase 2.0 predicted that IGFBP7 was a target gene of miR-25-3p. Subsequently, dual-luciferase reporter gene assay verified that miR-25-3p can target the 3'-UTR of IGFBP7. Toward this end, gene cotransfection for rescue assay was arranged, and we discovered that miR-25-3p can promote cell growth and repress cell apoptosis in TNF- $\alpha$-induced rat chondrocytes by targeting IGFBP7.

In conclusion, these data signified that miR-25-3p/IGFBP7 axis may possess a positive effect on mediating the pathologic response to apoptosis for chondrocytes. Furthermore, our study provides evidence that miR25-3p suppresses the apoptosis of rat chondrocytes and promotes cell proliferation by targeting IGFBP7. This study may be useful for future research on targeting miR-25-3p in the treatment of OA. However, there exists a limitation in our study. These findings were only observed in vitro and will be validated in an animal model in future experiments. An investigation with larger sample sizes are needed to provide a deeper insight regarding which pathways miR-25-3p modulates to affect the proliferation and apoptosis of OA chondrocytes. More and more methods have been shown to be effective in treating OA [33-35]. Therefore, it is still vital to explore new potential treatment strategies for OA progression.

\section{Acknowledgements}

Thanks for all the contributors and participants.

\section{Conflicts of interests}

The authors declare there is no conflict of interest regarding the publication of this paper.

\section{Funding}

This research was supported by the grants from Natural Science Foundation of Guangdong Province (No. 7301061).

\section{References}

1. Qiong J, Xia Z, Jing L, et al. Synovial mesenchymal stem cells effectively alleviate osteoarthritis through promoting the proliferation and differentiation of meniscus chondrocytes. Eur Rev Med Pharmacol Sci. 2020; 24(4): 1645-1655, doi: 10.26355/eurrev_202002_20338, indexed in Pubmed: 32141530.

2. Zou L, Liu J, Lu H. Influence of protein kinase RIPK4 expression on the apoptosis and proliferation of chondrocytes in osteoarthritis. Mol Med Rep. 2018; 17(2): 3078-3084, doi: 10.3892/mmr.2017.8209, indexed in Pubmed: 29257245.

3. Charlier E, Deroyer C, Ciregia F, et al. Chondrocyte dedifferentiation and osteoarthritis (OA). Biochem Pharmacol. 2019; 165: 49-65, doi: 10.1016/j.bcp.2019.02.036, indexed in Pubmed: 30853397.

4. Charlier E, Relic B, Deroyer C, et al. Insights on molecular mechanisms of chondrocytes death in osteoarthritis. Int J Mol Sci. 2016; 17(12), doi: 10.3390/ijms17122146, indexed in Pubmed: 27999417.

5. Yang Bo, Ni J, Long H, et al. IL- $1 \beta$-induced miR-34a up-regulation inhibits Cyr61 to modulate osteoarthritis chondrocyte proliferation through ADAMTS-4. J Cell Biochem. 2018; 119(10): 7959-7970, doi: 10.1002/jcb.26600, indexed in $\mathrm{Pu}-$ bmed: 29236314.

6. Wang X, Guo Y, Wang C, et al. MicroRNA-142-3p inhibits chondrocyte apoptosis and inflammation in osteoarthritis by targeting HMGB1. Inflammation. 2016; 39(5): 17181728, doi: 10.1007/s10753-016-0406-3, indexed in Pubmed: 27447821.

7. Miao G, Zang X, Hou H, et al. Bax Targeted by miR-29a regulates chondrocyte apoptosis in osteoarthritis. Biomed Res Int. 2019; 2019: 1434538, doi: 10.1155/2019/1434538, indexed in Pubmed: 30993110.

8. Duan ZX, Huang $\mathrm{P}$, Tu C, et al. MicroRNA-15a-5p regulates the development of osteoarthritis by targeting PTHrP in chondrocytes. Biomed Res Int. 2019; 2019: 3904923, doi: 10.1155/2019/3904923, indexed in Pubmed: 30949498.

9. Wu XF, Zhou ZH, Zou J. MicroRNA-181 inhibits proliferation and promotes apoptosis of chondrocytes in osteoarthritis 
by targeting PTEN. Biochem Cell Biol. 2017; 95(3): 437-444, doi: 10.1139/bcb-2016-0078, indexed in Pubmed: 28177757.

10. Li R, Wen Y, Wu B, et al. MicroRNA-25-3p suppresses epileptiform discharges through inhibiting oxidative stress and apoptosis via targeting OXSR1 in neurons. Biochem Biophys Res Commun. 2020; 523(4): 859-866, doi: 10.1016/j. bbrc.2020.01.050, indexed in Pubmed: 31954517.

11. Yao Y, Sun F, Lei M. miR-25 inhibits sepsis-induced cardiomyocyte apoptosis by targetting PTEN. Biosci Rep. 2018; 38(2), doi: 10.1042/BSR20171511, indexed in Pubmed: 29440462.

12. Ye C, Hou W, Chen Mo, et al. IGFBP7 acts as a negative regulator of RANKL-induced osteoclastogenesis and oestrogen deficiency-induced bone loss. Cell Prolif. 2020; 53(2): e12752, doi: 10.1111/cpr.12752, indexed in Pubmed: 31889368.

13. Benatar T, Yang W, Amemiya Y, et al. IGFBP7 reduces breast tumor growth by induction of senescence and apoptosis pathways. Breast Cancer Res Treat. 2012; 133(2): 563-573, doi: 10.1007/s10549-011-1816-4, indexed in Pubmed: 21997538.

14. Aki T, Hashimoto Ko, Ogasawara M, et al. A whole-genome transcriptome analysis of articular chondrocytes in secondary osteoarthritis of the hip. PLoS One. 2018; 13(6): e0199734, doi: 10.1371/journal.pone.0199734, indexed in Pubmed: 29944724

15. Gao ZQ, Guo X, Duan C, et al. Altered aggrecan synthesis and collagen expression profiles in chondrocytes from patients with Kashin-Beck disease and osteoarthritis. J Int Med Res. 2012; 40(4): 1325-1334, doi: 10.1177/147323001204000411, indexed in Pubmed: 22971484.

16. Kayal RA, Siqueira M, Alblowi J, et al. TNF-alpha mediates diabetes-enhanced chondrocyte apoptosis during fracture healing and stimulates chondrocyte apoptosis through FOXO1. J Bone Miner Res. 2010; 25(7): 1604-1615, doi: 10.1002/jbmr.59, indexed in Pubmed: 20200974.

17. Pearson MJ, Philp AM, Heward JA, et al. Long intergenic noncoding RNAs mediate the human chondrocyte inflammatory response and are differentially expressed in osteoarthritis cartilage. Arthritis Rheumatol. 2016; 68(4): 845-856, doi: 10.1002/art.39520, indexed in Pubmed: 27023358.

18. Li Y, Li S, Luo Y, et al. LncRNA PVT1 regulates chondrocyte apoptosis in osteoarthritis by acting as a sponge for miR488-3p. DNA Cell Biol. 2017; 36(7): 571-580, doi: 10.1089/ dna.2017.3678, indexed in Pubmed: 28520497.

19. Tan Li, Harper L, McNulty MA, et al. High-fat diet induces endoplasmic reticulum stress to promote chondrocyte apoptosis in mouse knee joints. FASEB J. 2020; 34(4): 5818-5826, doi: 10.1096/fj.201902746R, indexed in Pubmed: 32124494.

20. Chen J, Wu X. MicroRNA-103 contributes to osteoarthritis development by targeting Sox6. Biomed Pharmacother. 2019; 118: 109186, doi: 10.1016/j.biopha.2019.109186, indexed in Pubmed: 31302420.

21. Wang Xi, Ning Y, Zhou B, et al. Integrated bioinformatics analysis of the osteoarthritis-associated microRNA expression signature. Mol Med Rep. 2018; 17(1): 1833-1838, doi: 10.3892/ mmr.2017.8057, indexed in Pubmed: 29138855.

22. Coutinho de Almeida R, Ramos YFM, Mahfouz A, et al. RNA sequencing data integration reveals an miRNA interactome of osteoarthritis cartilage. Ann Rheum Dis. 2019; 78(2): 270-277, doi: 10.1136/annrheumdis-2018-213882, indexed in Pubmed: 30504444.
23. Li H, Bai B, Wang J, et al. Identification of key mRNAs and microRNAs in the pathogenesis and progression of osteoarthritis using microarray analysis. Mol Med Rep. 2017; 16(4): 5659-5666, doi: 10.3892/mmr.2017.7251, indexed in Pubmed: 28849222.

24. Yu C, Chen WP, Wang XH. MicroRNA in osteoarthritis. J Int Med Res. 2011;39(1): 1-9, doi: 10.1177/147323001103900101, indexed in Pubmed: 21672302.

25. Kolhe R, Hunter M, Liu S, et al. Gender-specific differential expression of exosomal miRNA in synovial fluid of patients with osteoarthritis. Sci Rep. 2017; 7(1): 2029, doi: 10.1038/ s41598-017-01905-y, indexed in Pubmed: 28515465.

26. Wang M, Yang YOu, Jin Q, et al. Function of miR-25 in the invasion and metastasis of esophageal squamous carcinoma cells and bioinformatical analysis of the miR-106b-25 cluster. Exp Ther Med. 2018; 15(1): 440-446, doi: 10.3892/ etm.2017.5358, indexed in Pubmed: 29250158.

27. Rao HC, Wu ZK, Wei Sd, et al. MiR-25-3p serves as an oncogenic MicroRNA by downregulating the expression of merlin in osteosarcoma. Cancer Manag Res. 2020; 12: 8989-9001, doi: 10.2147/CMAR.S262245, indexed in Pubmed: 33061594.

28. Wu T, Hu H, Zhang T, et al. miR-25 Promotes Cell Proliferation, Migration, and Invasion of Non-Small-Cell Lung Cancer by Targeting the LATS2/YAP Signaling Pathway. Oxid Med Cell Longev. 2019; 2019: 9719723, doi: 10.1155/2019/9719723, indexed in Pubmed: 31316723.

29. Chen H, Pan H, Qian Yi, et al. MiR-25-3p promotes the proliferation of triple negative breast cancer by targeting BTG2. Mol Cancer. 2018; 17(1): 4, doi: 10.1186/s12943-017-0754-0, indexed in Pubmed: 29310680.

30. Chen D, Siddiq A, Emdad L, et al. Insulin-like growth factor-binding protein-7 (IGFBP7): a promising gene therapeutic for hepatocellular carcinoma (HCC). Mol Ther. 2013; 21(4): 758-766, doi: 10.1038/mt.2012.282, indexed in Pubmed: 23319057.

31. Zhang Le, Lian R, Zhao J, et al. IGFBP7 inhibits cell proliferation by suppressing AKT activity and cell cycle progression in thyroid carcinoma. Cell Biosci. 2019; 9: 44, doi: 10.1186/ s13578-019-0310-2, indexed in Pubmed: 31183073.

32. Verhagen HJ, de Leeuw DC, Roemer MGm, et al. IGFBP7 induces apoptosis of acute myeloid leukemia cells and synergizes with chemotherapy in suppression of leukemia cell survival. Cell Death Dis. 2014; 5: e1300, doi: 10.1038/cddis.2014.268, indexed in Pubmed: 24967962.

33. Yang Q, Zhou Y, Cai P, et al. Downregulation of microRNA-23b-3p alleviates IL- $1 \beta$-induced injury in chondrogenic CHON-001 cells. Drug Des Devel Ther. 2019; 13: 2503-2512, doi: 10.2147/DDDT.S211051, indexed in Pubmed: 31440033 .

34. Yu Y, Zhao J. Modulated Autophagy by MicroRNAs in osteoarthritis chondrocytes. Biomed Res Int. 2019; 2019: 1484152, doi: 10.1155/2019/1484152, indexed in Pubmed: 31205933.

35. Jeon OkH, Wilson DR, Clement CC, et al. Senescence cell-associated extracellular vesicles serve as osteoarthritis disease and therapeutic markers. JCI Insight. 2019; 4(7), doi: 10.1172/ jci.insight.125019, indexed in Pubmed: 30944259.

Submitted: 29 October, 2020 Accepted after reviews: 28 January, 2021 Available as AoP: 12 February, 2021 